Historic, Archive Document

Do not assume content reflects current scientific knowledge, policies, or practices. 



\section{HERBST BROTHERS}

Established 1876

\section{SEEDMEN and PLANT BROKERS}

\section{South Street}

New York, N. Y.

\section{Price List}

(THIS LIST CANCELS ALL PREVIOUS QUOTATIONS)

(Subject to change)

\section{Terms of Sale}

We give for ourselves or our principals no warranty, express or in:plied as to description, quality or productiveness or any other matter of the seed, plants or bilbs we responsible for the crop in

PAYMENT: ON SEED net cash less $5 \%$ for unestabllshed accounts; others less $5 \%$ if paid within ten days or 60 days net. ON NURSERY STOCK to be arranged at time of sale.

PRICES are for net weights, packing extra at cost.

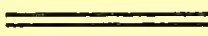

\section{New Crop Seed}

\section{Genuine Imported}

\section{Ulmus Pumilia \\ Dwarf Asiatic Elm}

As the seed is of very low viability we import from the

Orient only a small excess over the quantities needed

for filling advance orders. We shall be glad to have

FOB New York per lb. net

your order while this surplus remains available....... $\$ 2.25$

\section{Also Order at once Your lleeds of:}

ULMUS americana, American Elm .............. .75

"campestris, English Elm ................. 1.25

“ japonica, Japanese Elm ............... 1.75

ACER dasycarpum, Silver Maple ............... .50

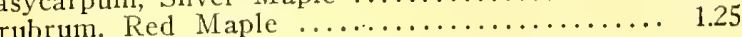

\section{Convention Notice}

On your way to or from the BOSTON CONVENTION look us up. A hearty welcome will be awaiting you at 42 South St.

At the Convention Gus and Fred Herbst will be on the floor to execute your orders to buy or sell Nursery Stock. Let us know what you have to trade.

\section{Japanese Flowering Cherries}

The very best results are obtained only when the seed is sown immediately after harvest. We will bring your orders forward from Japan just as soon as the seed ripens and can be conditioned. As in the case of Ulmus pumila we do not import much in excess of advance orders. We offer only genuine imported Japanese and Chinese seed as follows:

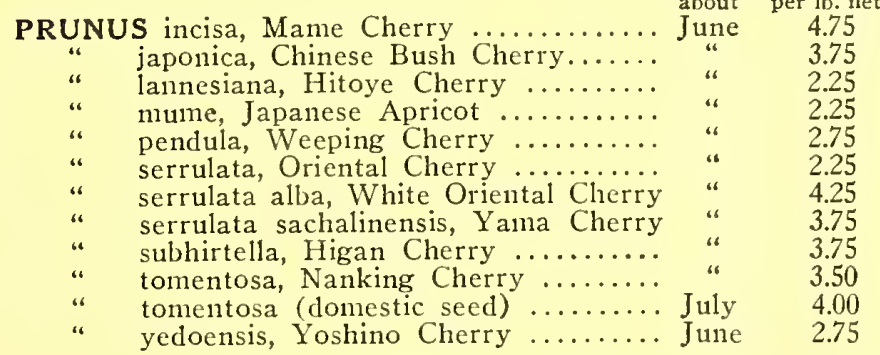




\section{Other Items We Recommend To Your Attention How}

Early maturing seeds are generally delicate in structure and of low vitality The laws of Nature generally require that they be planted soonest vitality. The laws of Nature generally require that they be planted soonest
possible after liarvest. Let us have your order now so that we can ship possible after larvest. Let us have your
them to you immediately after collection.

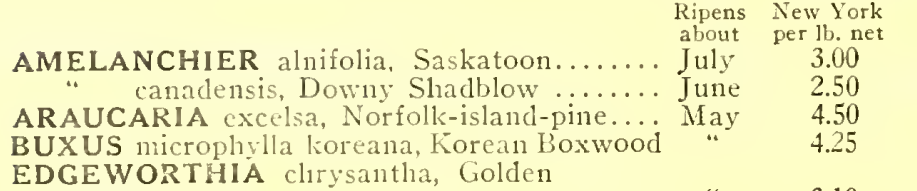

EDGEWORTHA clirysantha, Golden ...... " 3.10

ELAEAGNUS macrophylla, Bloodleaf

Elaeagnus ......................... June 2.75

ELAEAGNUS longipes, Cherry Elaeagnu..... " J" 3.00

ERIOBOTRYA japonica, Loquat ............ A April 2.25

." japonica, Nammoth Fruiting Loquat. April 2.75

FATSIA japonica, Fatsia ................................ 3.25

HEDERA helix, English Ivy .................. 1.00

MORUS alba, White Mullberry .............. May 2.55 alba, tatarica, Russian Muberry......." 2.55

PAEONIA chincnsis .................... Aug. $\quad 5.00$
" obovata ................... 5.00
suffruticosa (Montan) Tree Peony... “ " 7.00
SAMBUCÚS racenosa, European Red Elder. June 2.25

VIBURNUM odoratissina, Sweet Viburnum. . Aug. 2.25

\section{General Stock \\ Fruit and Economic Plants}

JUGLANS manchurica, Manchurian Walnut........ .75

PYRUS serotina, Japan Pear ................... 5.00

PYRUS ussuricnsis, Ussurian Pear ............... 7.00

\section{Conifers}

ABIES

FOB New York

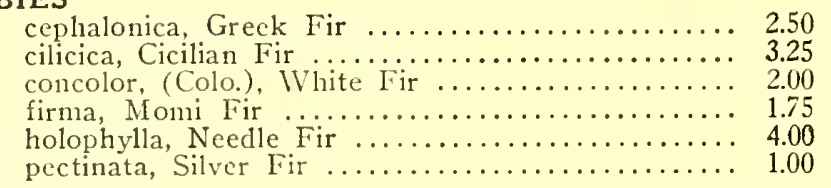

BIOTA orientalis (sec Thuja orientalis)

CEDRUS atlantica, Atlas Cedar .............. 3.25

libani, Cedar of Lebanon ........................... 3.50

CEPHALOTAXUS koraiana ....................... 1.75

CHAMAECYPARIS lawsoniana, Lawson Cypress.... 2.50

obtusa, Hinoki Cypress ..................... 2.25

CRYPTOMERIA japonica, Common Cryptomeria.... 1.10

CUPRESSUS arizonica, Arizona Cypress.......... 3.50 macrocarpa, Monterey Cypress ................. 2.50

sempervirens ....................... 2.50

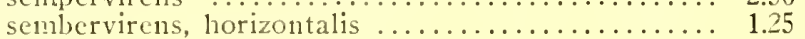

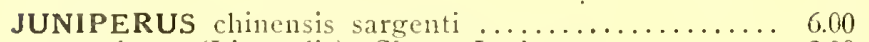

conferta (Littoralis), Shore Juniper..................... 2.00

scopulorum, (Colo.), Colorado Juniper ............. 1.65

virginiana, Red-cedar (Southern).................... 1.15

LARIX curopaca, European Larch .............. 1.60

LIBOCEDRUS decurrens .................. 3.75
PICEA canadensis, White Spruce

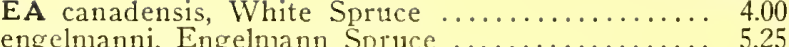

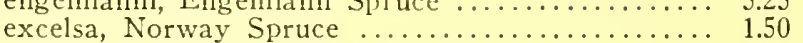

glauca albertiana, Black Hills Spruce ............... 6.50

jezoensis, Yeddo Spruce ........................ 6.00

pungens, Colorado Spruce .................. 5.45

sitchensis, Sitka Spruce ..................... 6.00

PINUS aristata, Bristlecone Pine ............... 3.50

attentuata, Knobcone Pine ...................... 6.00

banksiana, Jack Pine ...................... 3.25

bungeana, Lacebark Pine .................... 3.25

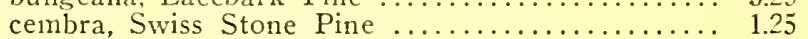

densiflora, Japanese Red Pine ................. 1.75

edulis, Nut Pine ............................ $\quad .90$

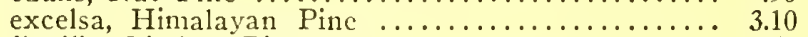

flexilis, Limber Pine .......................... 3.50

jeffreyi, Jeffrey Pine .................... 2.75

montana, Swiss Mountain Pine ............... 2.50

montana nughus, Mugho Pine .................. 4.10

montana uncinata, Large Swiss Pine............ 3.00

monticola, Western White Pine ................ 6.50

nigra, Austrian Pine ........................ 1.75

nigra poiretiana, Corsican Pine ............... 3.40

palustris, Longleaf Pine ................... 3.00

parviflora, Japanese White Pine ................... 3.50

pentaphylla $\ldots \ldots \ldots \ldots \ldots \ldots \ldots \ldots \ldots \ldots \ldots . .3 .50$

ponderosa, Western Yellow Pine ............... 2.00

radiata, Monterey Pine ....................... 3.25

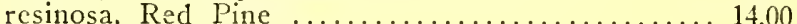

rigida, Pitch Pine ....................... 6.00

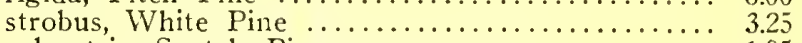

sylvestris, Scotch Pine ..................... 1.95

tabula eformis, Chinese Pine ............... 4.25

taeda, Loblolly Pine ....................... 5.00

thunbergi, Japanese Black Pine ................. 1.80

SCIADOPYTIS verticellata, Unbrella Pine.......... 3.75

TAXUS baccata, English Yew ................ 2.25

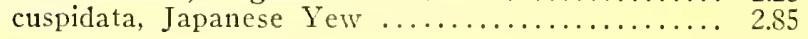

THUJA

orientalis, Oriental Arborvitae ............. 1.20

plicata, Giant Arborvitae ........................ 6.00

TSUGA sieboldi, Sicbold Hemlock ............. 5.30

\section{Deciduous and Evergreens}

FOB New Yosk per lb. ne

ACER palmatum, Japanese Maple ............. 2.90 palmatum, atropurpureum, Japanese

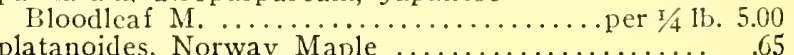

AILANTHUS glandulosa, Ailanthus .......... .90

ALBıZZıA julibrissin, Silktrec ............... 2.90

AMPELOPSIS quinquefolia, (Virginia Creeper) Berries 1.75 tricuspidata (veitchi), Japanese C. Clean Seed.... 1.50

ARALIA spinosa, Devils-walkingstick ........... 2.50 ARCTOSTOPHYLOS uva ursi, (Berrics) .......... 1.85

ARONIA arbutifolia, Red Chokeberry ............. 1.45

AUCUBA japonica, Japanese Aucuba ............. 2.10

AZALEA kurume, mixed colors ............ per oz. 20.00 poukhanensis .......................... oz. 8.00 schlippenbachi $\quad$.............. peroz. $\$ 3.00$ per $1 / 4$ lb. 10.00

BETULA alba, European White Birch ........... .95

BIGNONIA radicans, Trumpetcreeper ............ 4.00 CALYCANTHUS florida, Common Swectshrub ........ 1.55

CARAGANA arborescens, Siberian Pea-trce........ 1.85 


\section{Flower Seed}

CARPINUS betulus, European Hornbeam.......... 90

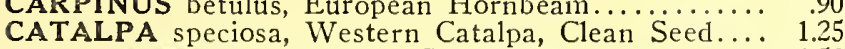

CINNAMOMUM camphora, Camphor Tree ......... 1.50

COLUTEA arborescens, Common Bladder-senna....... 1.00

CORNUS mas, Cornelian-cherry ................. 1.00 paniculata, Gray Dogwood ........................ 1.10

sanguinea, Bloodtwig Dogwood ............... $\quad .85$

COTONEASTER acutifolia, Peking Cotoneaster ....... 2.15 microphylla, Rockspray ...................... 2.25

CRATAEGUS nitida, Glossy Hawthorn, Berries..... 1.20 oxyacantha, English Hawthorne, (two stone) C.S. 2.25

ELAEAGNUS angustifolia, Russian Olive ......... 95 umbellata, Autumn Elaeagnus ................ 3.15

EUONYMUS europaeus, European Burningbush ....... 1.25 japonicus, Evergreen Burningbush ............... 2.45 radicans, Wintercreeper .................. 2.25

EXOCHORDiA grandiflora, Common Pearlbush C.. S. 4.75

FATSIA japonica, Fatsia ................ 3.45

FRAXINUS americana, White Ash ............... 8.85 mandschurica, Manchurian Ash ............... 1.85 ornus, Flowcring Ash .................... ${ }^{85}$

GLEDITSIA triacanthos, Common Honeylocust ................75

HALESIA tetraptera, Great Silverbcll ............ 1.75 HAMAMELIS virginiana, Common Witch-hazel ...... 1.75

HEDERA helix, English Ivy ......................... HIBISCUS

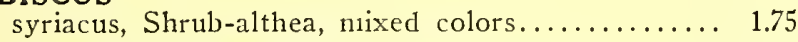

ILEX aquifolium, English Holly ................ 2.10 crenata, Japanese Holly ................... 2.75

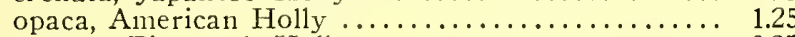
serrata, Finetooth Holly ................... 3.25 verticillata, Common Winterberry .............. 1.25

LABURNUM vulgare, Goldenchain ............. 1.45

LIGUSTRUM ibota $. . \ldots \ldots \ldots \ldots \ldots \ldots \ldots \ldots \ldots \ldots \ldots \ldots \ldots . .75$

MAHONIA aquifolium, Oregon Hollygrape ......... 3.25

MELIA azedarach, Chinaberry ................... 1.25

MORUS alba tatarica, Russian Mulberry ........... 2.00

NYSSA sylvatica $\ldots \ldots \ldots \ldots \ldots \ldots \ldots \ldots \ldots \ldots \ldots . \ldots \ldots . \ldots \ldots$

PAULOWNIA tomentosa, Royal Pawlinia .......... 1.75

PLATANUS occidentalis, American Planetree ........ .75

RHAMNUS frangula $\ldots \ldots \ldots \ldots \ldots \ldots \ldots \ldots \ldots \ldots . \ldots \ldots$ RHODODENDRON metternichi ............... per oz. 1.50 RHUS glabra, Smooth Glabra ................. .70

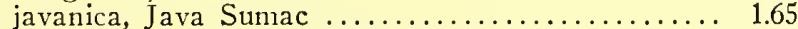

ROBINIA pseudoacacia, Common Locust ........... .85

ROSA lucida, Virginia Rose ................... 1.10

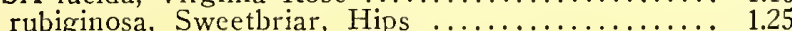

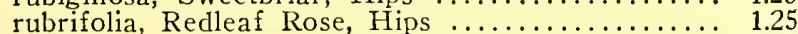

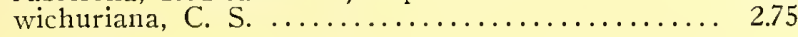

SAMBUCUS racemosa, red $\ldots \ldots \ldots \ldots \ldots \ldots \ldots \ldots . \ldots \ldots \ldots .10$

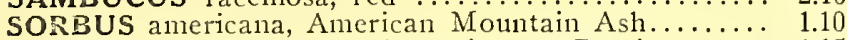
aucuparia, European Mountain-ash, Bcrries ....... 1.15

SYRINGA dilatata, Clean Seed ................ 12.00 vuigaris, Common Lilac, Clean Seed............. 4.00

TAMARIX gallica, French Tamarix ........... 2.00

TILIA platyphyllos, Bigleaf European Linden........ 1.15 tomentosa, Silver Linden ................. 1.25

VIBURNUM lantana, Wayfaring-tree ........... 1.45 opulus, European Cranberrybush, Berries ........ 1.25 sargenti, Sargent Cranberrybush ............... 3.25 sieboldi, Siebold Viburnum .................... 4.00 tomentosum, Doublefile Viburnum ............... 3.25

WISTARIA sinensis, blue $\ldots \ldots \ldots \ldots \ldots \ldots \ldots \ldots .6 \ldots$

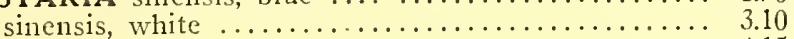
sinensis, multijaga blue $\ldots \ldots \ldots \ldots \ldots \ldots \ldots \ldots . . .15$

ZELKOVA serrata, Sawleaf Ze!kova ............ 2.75

\section{ACONITUM}

FOB New York

fisheri, blue ..................... \$1.00 $\$ 3.00$

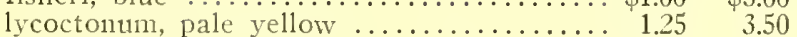

japonicum, bright blue ................. $1.50 \quad 4.00$

\section{ADONIS}

aestivalis

\section{AGERATUM}

mexican tall mixed ..................

nanum blue perfection ................. 40

.60

1.00

\section{AGROSTEMMA}

coronaria

coronaria red, (Rose Champion) .................

\section{.70}

\section{AMPELOPSIS}

(See list of Deciduous and Evergrecn seeds)

\section{ANCHUSA}

capensis, (dark blue) ................ . . 25 70

italica grfl., Dropmore ................. $.35 \quad 1.00$

italica grfl., Opal ................... .75 2.00

ANEMONE

cernua, blackish velvety purple ......... $\quad .70$

2.00

AQUILEGRA (Columbine)

sgle. fl. mxd. ....................... 75

dble. fl. mxd. .

choice lg. spurred hybrids mxd.......... 3.00

2.50

4.00

\section{BELLIS}

perennis dble, fl. mxd.

3.00

BOCCONIA

cordata

CALLIOPSIS

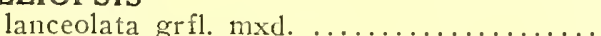

CAMPANULA (Bell Flower)

medium, (Canterbury Bells) sgle. mxd...... medium, (Canterbury Bells) dble. mxd..... pyramidalis (Chimney Bell Flower) blue.... speculum (Venus' Looking Glass), mxd...

\section{CANDYTUFT (Iberis)}

annual, mixed

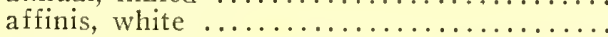

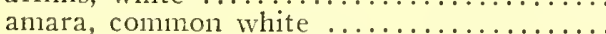

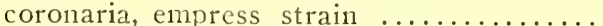

coronaria, giant hyacinth fl. ................

coronaria, white rocket $\ldots \ldots \ldots \ldots \ldots \ldots . . . . . .$.

\section{CELOSIA}

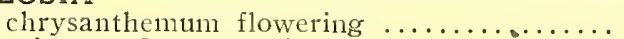

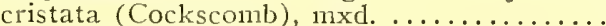
pyramidalis plumosa $m x d . . . \ldots \ldots \ldots \ldots$.

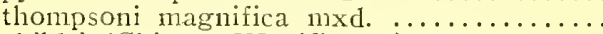

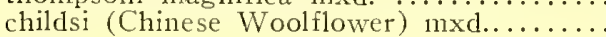

\section{CHRYSANTHEMUM}

coronarium, dble. mxd. ..............

leucanthenum, Spring Marguerite ........ leucanthenum, hybr. Shasta Daisy ....... leucanthemum, hybr. Alaska ...............

\section{CIMICIFUGA}

simplex

\section{CLEMATIS}

paniculata

COIX

lachrymae (Job's Tear) ................. . 25

\section{cosmos}

carly fl. mxd.

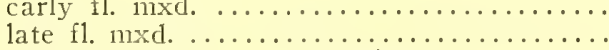

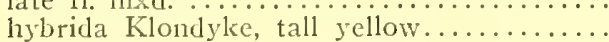

$\ddot{90}$ 
DAHLIA

FoB New York
per oz. per $1 /$ it

variabilis, dbde, mxd.

variabilis, eactus fl. nixd. ................... $3.00 \quad 8.00$

DELPHINIUM (see also Larkspur)

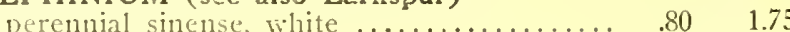

DIANTHUS

caryophyllus (Carnation), sgle. mxd........ .40 1.10

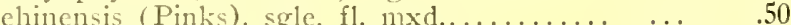

chinensis (Pink), dlple. fl. mxd...............
plumarius (Grass Pinks), sgle. fl. mxd....

DIGITALIS (Foxglove)

purpurea gloxinaeflora lg. f1. nuxd......... $35 \quad 90$

In eolors, purple and white.............. $.40 \quad 1.00$

EUPHORBIA

heterophvlla (Fire on the Mountain)...... .60 .60

variegata (Snow on the Mountain)........... $.25 \quad .60$

EURALIA (Miscanthus)

japoniea, Japanese Euralia ...............5 50

GAILLARDIA

picta lorenziana, dble. mxd, ............. . .25 .65

grfl. lo. fl. $117 x d, \ldots \ldots \ldots \ldots \ldots \ldots \ldots, .30 \quad .75$

GYPSOPHILA

elegans, white

elerans, atrosanguinea erimson

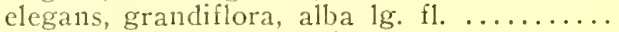

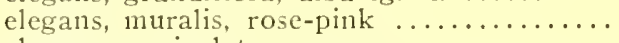

elegans, panieulata ...........

HELICHRYSUM (Strawflower)

monostrosum, dble. lg. fl. $11 x \mathrm{xd} \ldots \ldots \ldots \ldots \ldots, .30 \quad .85$

HELIOTROPIUM

1g. fl. dble, $m x d \ldots . . . \ldots \ldots \ldots \ldots \ldots . . .25$

HESPERIS

matronalis

HOLLYHOCK

sgle. fl. nuxd.
dble. fl. nuxd.

IPOMOEA

IRIS

eardinalis (Cardinal Climber) ............. 1.40

ensata $\ldots . .50 \quad 8.00$

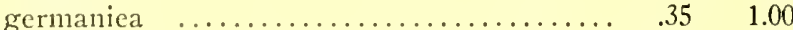

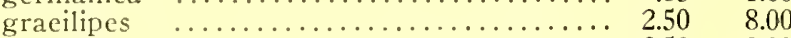

japonica $\ldots \ldots \ldots \ldots \ldots \ldots \ldots \ldots \ldots \ldots \ldots .2 .50 \quad 8.00$

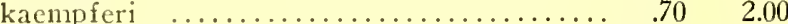

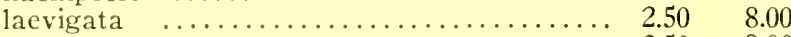

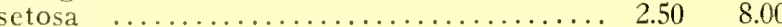

sibiriea $\ldots \ldots \ldots \ldots \ldots \ldots \ldots \ldots \ldots \ldots \ldots \ldots, 1.00 \quad 3.00$

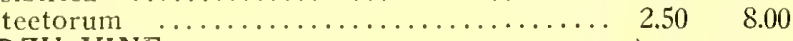

KUDZU VINE

pueraria thunbergi $\ldots . \ldots \ldots \ldots \ldots \ldots \ldots \ldots \ldots . . . .65$

LARKSPUR

tall dble. mxd. ....................... .40 $\quad .90$

LIATRIS

pysnostachya $\ldots \ldots \ldots \ldots \ldots \ldots \ldots \ldots \ldots, 1.00 \quad 3.00$

LILIUM

Duratum

cernum ......................... "

cordifolium ....................... “

elegans, orange .................... “ “ 1.00

giganteum ................... " 2.00

henryi ........................ " 3.00

japonieum, pink ..................... "

medeoloides ..................... "

melpomene ........................ "

platyphyllum (Macranthum) ............ “ 1.00

regale

rubellum ....................... " 1.25

tenuifolium

ventusum fujihirato ................. " 2.00

LOBELIA

erinus, nuxd. ........................... .50 1.30

eriulus, blue . ............................... 40

erinus, white .......................... 1.50

LOTUS

speciosum, mixd. ................... .50 1.50

In Colors; pink and white.............. $75 \quad .00$

\section{LUPINUS}

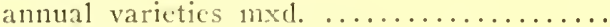

polyphyilus perennial mxe. ..............

nolyphyllus perennial white
MIMOSA

pudiea (Sensitive Plant)

MOMORDICA

eharantia (Balsam Pear)

MYOSOTIS (Forget-me-not)

palustris, blue $\ldots \ldots \ldots \ldots \ldots \ldots \ldots \ldots . . .60 \ldots \ldots$

NICOTIANA (Flowering Tobacco)

affinis, white, night scented............. .25 .60

PETUNIA

hybr. finest $\operatorname{mxd} . \ldots \ldots \ldots \ldots \ldots \ldots \ldots \ldots .60 .60 \ldots$

hybr. alba pure white ................ $\quad .70 \quad 1.70$

hybr. Carmen Sylva, violet white throat... $\quad .70 \quad 1.70$

hybr. Howard Star .................. $.80 \quad 2.00$

hybr. Lord Courtney, bril. earminc....... $\quad .95 \quad 2.70$

hybr. maculata (Striped and Blotehed).... $\quad .80 \quad 2.00$

hybr. compacta, Rose of Heaven ......... $1.20 \quad 3.50$

hybr. compacta, Rosy Morn ................ $1.50 \quad 4.00$

hybr. grfl. lg. fl. mxd................ 3.2510 .00

PHLOX

drummondi cuspidata (Star Phlox) nxd.... $.75 \quad 1.85$

drummondi grfl. lg. fl. mxd. ........... $65 \quad 1.45$

PHYSALIS (Chinese Lantern Plant)

francheti

PLATYCODON

grandiflorum blue $\ldots \ldots \ldots \ldots \ldots \ldots \ldots \ldots . .90 \quad 2.50$

POPPY

orientale (Oriental Poppy) ............. . .85 2.00

PRIMULA

japonica $\operatorname{mxd} \ldots \ldots \ldots \ldots \ldots \ldots \ldots \ldots \ldots, 1.60 \quad 5.00$

SALVIA

splendens

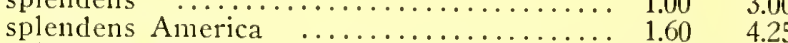

splendens Bonfire .................... $1.45 \quad 3.80$

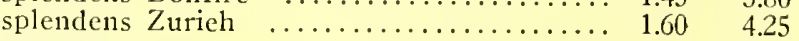

\section{SCABIOSA}

annual tall dble. $\operatorname{mxd} \ldots \ldots \ldots \ldots \ldots \ldots \ldots \ldots . .25 \quad .65$

perennial japonica ..................... $.45,1.05$

STOCKS

tall $\mathrm{lg}$. fl. varieties $\operatorname{mxd} . \ldots \ldots \ldots \ldots \ldots \ldots . .45 \quad .1 .10$

SUNFLOWER

hybridus red, selected strain............. ... $\quad .50$

eueumerifolius, Miniature Sunflower ..... ... $\quad .60$

SWEET WILLIAM

sgle. fl. $\operatorname{mxd} . \ldots \ldots \ldots \ldots \ldots \ldots \ldots \ldots \ldots \ldots, .25 \quad .45$

dble. fl. mxd. ...................... 35

TORENIA

fournicri blue $\ldots \ldots \ldots \ldots \ldots \ldots \ldots \ldots \ldots . \ldots .2 .25 \quad 7.00$

VERBENA

hybr. mxd. . ..................... .60 1.50

VINCA

rosea mxd.

rosea rose

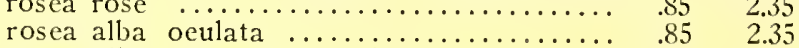

ZEA

rosea alba pura, pure white ............ $.85 \quad 2.35$

japoniea, Japanesc Rainbow Corn ........... . . 30

ZINNIA

elegans robusta giants $\operatorname{mxd} \ldots \ldots \ldots \ldots \ldots . .50 \quad 1.25$

Colors: burns orange golden yellow

purple

rose queen

searlet

white

clegans pumila, dwarf dble. mxd........... $.35 \quad .85$

dahlia flowered, extra lg. mxd........... . .85 2.50

Colors: erimson-monareh]

exquisite
meteor
old rose
polar bear
searlet flame

1.40 